\section{Narrow-channel-spacing 32-channel arrayed- waveguide-grating multiplexer with an innovative alignment method}

\author{
Jian Li \\ Junming An \\ Junlei Xia \\ Hongjie Wang \\ Xiongwei $\mathrm{Hu}$ \\ The Chinese Academy of Science \\ Institute of Semiconductor \\ R\&D Center of Optoelectronics \\ Beijing 100083, China \\ E-mail: lijian@red.semi.ac.cn
}

\begin{abstract}
A 32-channel $50-\mathrm{GHz}$ spaced arrayed-waveguide grating with our innovative configuration has been designed and fabricated. The performance of the device has been fully tested by using a tunable laser light source, optical power meter, and polarization controller. The insertion loss (IS) of the device is $4.2 \sim 7.4 \mathrm{~dB}$. The crosstalk is about $-28 \mathrm{~dB}$. The IS uniformity is less than $3.2 \mathrm{~dB}$. With our configuration, the performance of the device has been enhanced effectively and the difficulty in alignment process has been decreased obviously. (C) 2005 Society of Photo-Optical Instrumentation Engineers. [DOI: 10.1117/1.1925048]
\end{abstract}

Subject terms: optical planar waveguides; phased arrays; wavelength division multiplexing.

Paper 050029LR received Jan. 18, 2005; revised manuscript received Mar. 28, 2005; accepted for publication Apr. 5, 2005; appeared online Apr. 11, 2005; published online Jun. 7, 2005.

The explosive growth in demand for Internet services and data communications has prompted many research groups to study dense wavelength division multiplexing (DWDM) systems with narrow channel spacing. ${ }^{1-3}$ Arrayedwaveguide gratings (AWG) fabricated using silica-based waveguides play a key role as practical multi/ demultiplexers in these narrow-channel-spacing DWDM systems owing to their good stability, good channel uniformity, high wavelength accuracy, and low cost. These features are obtained by employing planar lightwave circuit (PLC) technology. ${ }^{4}$

The design parameters of our AWG are shown in Table 1. The channel spacing and the number of channels are 50 $\mathrm{GHz}$ and 32, respectively. To achieve these values, we calculate that the geometrical path length difference between adjacent arrayed waveguides should be $128.418 \mu \mathrm{m}$.

The device's characteristics are tested by the tunable laser light source, optical power meter, and polarization controller. The AWG chip is illuminated by the laser beam from a standard cone-shaped single-mode fiber and followed by a standard $127-\mu \mathrm{m}$ spaced single-mode fiber array. The polarization dependent loss (PDL) is tested by the method of Muller matrix. The end face of the AWG chip and the fiber array are polished without coating.

Figure 1 shows the photograph of the AWG device with the parameters listed in Table 1, which we designed with our innovative configuration. Compared to the conventional configuration, the minimum radius of curvature waveguide is increased to $7.3 \mathrm{~mm}$ from the $4.3 \mathrm{~mm}$ in a diminished chip size, and this change will decrease the chip's insertion loss observably.

Figure 2 shows the schematic diagram of the alignment process of the AWG device. By adding a Y-branch waveguide to the AWG and arranging the input/output channel in a neat row, the uniformity distance between each waveguide in the output side is $127 \mu \mathrm{m}$ strictly to fit for the fiber array's spacing. Even for the $N \times N$ multi/demultiplexer, the device only needs to be polished on one side and the whole configuration could be aligned and packaged using one fiber array. The alignment process is divided into two steps. The first is accurately aligning the single-mode fiber to the input port of the Y-branch waveguide. When the infrared CCD camera receives the two clearly lighted spots on the output side of the Y-branch, the first step is over and we hold the fiber's position. The second step is aligning the fiber array to the output ports of the Y-branch waveguide. When the optical power which is output from the Y-branch reaches the maximum, the AWG chip has already accurately aligned to the fiber array simultaneous. Then we can move the single-mode fiber and connect the multiwavelength light source to the input port of the AWG and finish our testament process. With our innovative configuration, the single-mode fiber is easily aligned to the waveguide and only one fiber array would align to the AWG device accurately by using the 6-axis movement stage. The process time of polishing and alignment can be decreased obviously.

The transmission spectrum in the 1550 -nm windows is shown in Fig. 3 where the input port 2 is used for the measurement. The measured $3-\mathrm{dB}$ bandwidth is $0.19 \mathrm{~nm}$. The output power uniformity over the 32 channels is less than $3.2 \mathrm{~dB}$. The insertion loss of the AWG is about 4.2 7.4 dB. The crosstalk is about $-28 \mathrm{~dB}$. The insertion loss of the center output waveguide is $4.2 \mathrm{~dB}$. Reference waveguides running alongside the AWG show a loss of 2 $\mathrm{dB}$, which include fiber coupling loss, bending loss, and

Table 1 Design parameters of a $32 \times 0.4-n m$ AWG.

\begin{tabular}{ll}
\hline Number of channels & 32 \\
Central wavelength $(\mu \mathrm{m})$ & 1.5525 \\
Channel spacing $(\mathrm{nm})$ & $0.4(50 \mathrm{GHz})$ \\
Core size $\left(\mu \mathrm{m}^{2}\right)$ & $6 \times 6$ \\
Free spectral range $(\mathrm{nm})$ & 12.88 \\
Diffraction order & 120 \\
Free propagation range $(\mu \mathrm{m})$ & 9419.72 \\
Path length difference $\Delta L(\mu \mathrm{m})$ & 128.418 \\
Number of arrayed waveguides & 130 \\
\hline \hline
\end{tabular}




\section{E}

L

E

T

$\mathrm{T}$

E

$\mathbf{R}$

S

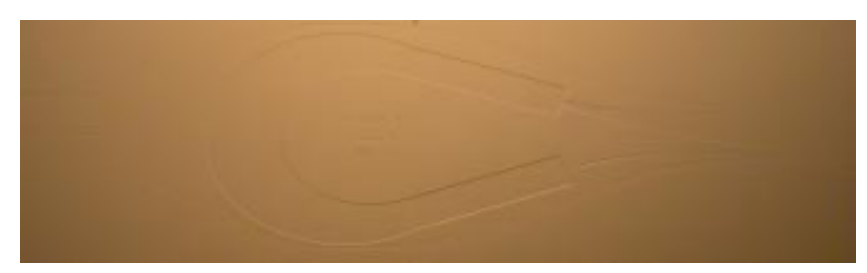

Fig. 1 Photograph of AWG designed with our configurations.

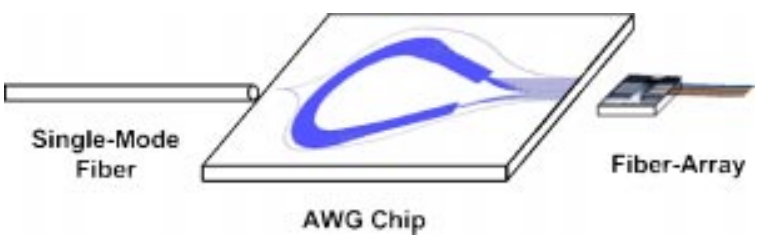

Fig. 2 Schematic diagram of the alignment process.

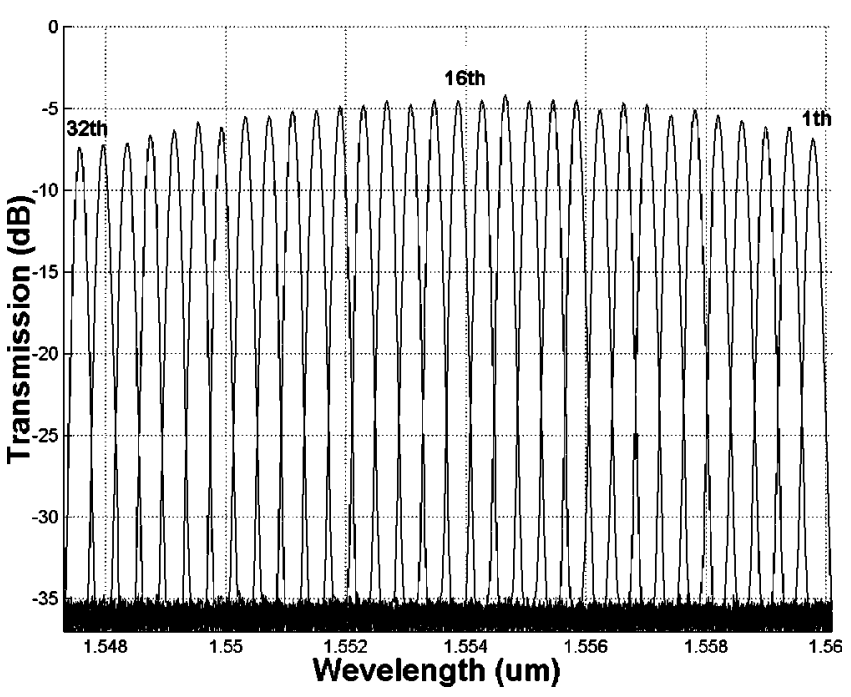

Fig. 3 Transmission spectrum of 32 channels.

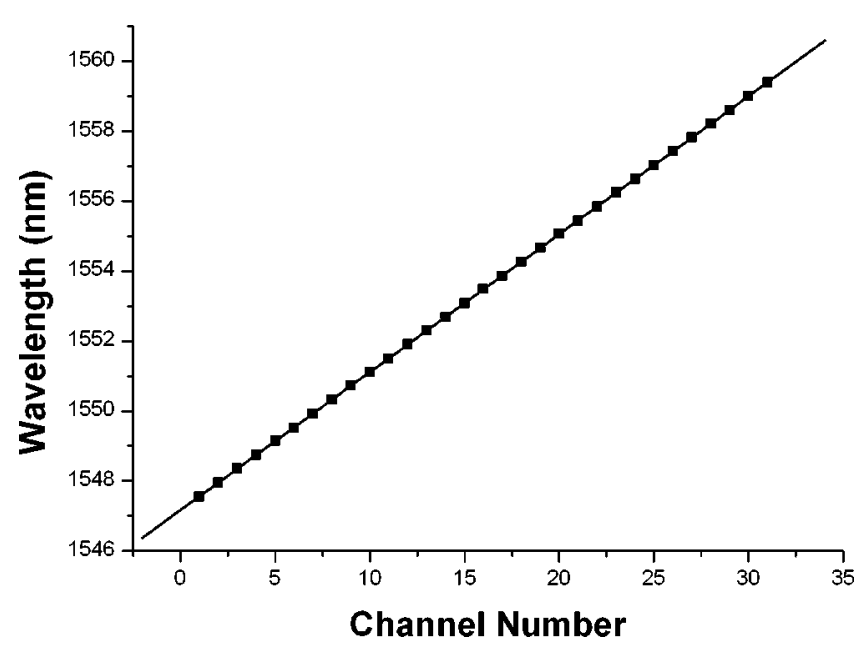

Fig. 4 Measured pass wavelengths of the multiplexer output channels. The slope is $0.39419 \mathrm{~nm} /$ channel.

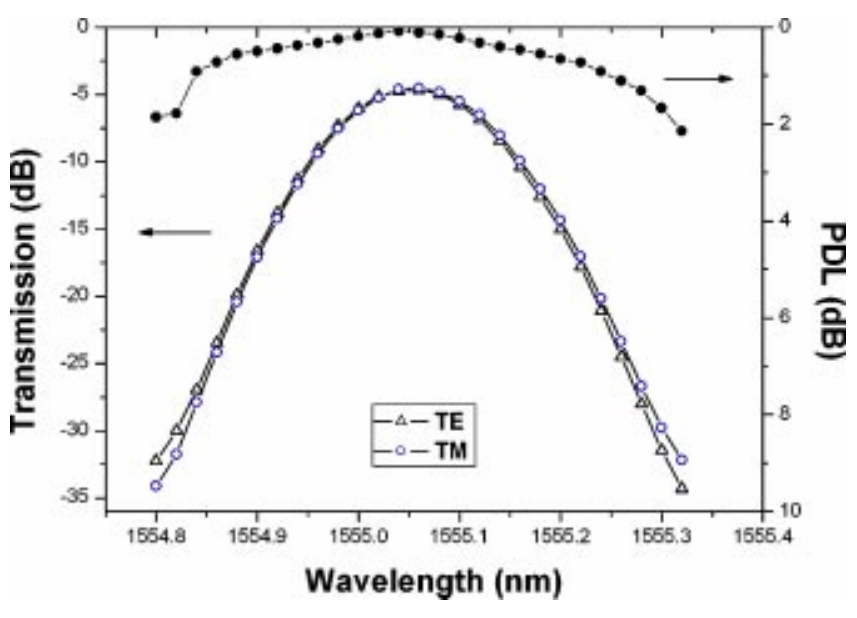

Fig. 5 Measured polarization dependence loss.

propagation loss. So the additional loss of the device is 2.2 $\sim 5.4 \mathrm{~dB}$.

Figure 4 shows the measured pass wavelengths of the AWG multiplexer. The slope is $0.39419 \mathrm{~nm} / \mathrm{channel}$. It is almost the same with the designed spacing of $0.4 \mathrm{~nm}$.

Figure 5 shows the test result of the chip's PDL, which is less than $0.4 \mathrm{~dB}$ in the $3-\mathrm{dB}$ bandwidth. Data show that the TE mode and the TM mode are almost in the same position.

In conclusion, we have designed a 50-GHz spaced 32channel single-side alignment AWG. The device's insertion loss is $4.2 \sim 7.4 \mathrm{~dB}$; the crosstalk is less than $-28 \mathrm{~dB}$; the PDL is less than $0.4 \mathrm{~dB}$ in $3-\mathrm{dB}$ bandwidth and the IS uniformity is less than $3.2 \mathrm{~dB}$. By using our innovative configuration, the polishing and alignment process time can be decreased obviously and this will decrease the cost in AWG fabrication. Compare to the conventional configuration, the AWG's minimum radius of curvature waveguide is increased to $7.3 \mathrm{~mm}$ from the $4.3 \mathrm{~mm}$ in a diminished chip size, and this change will enhance the performance of the AWG device observably.

\section{Acknowledgments}

This project is supported by National Basic R\&D Plan of China (No. G2000036602) and National Natural Science foundation of China (No. 60477035).

\section{References}

1. K. Okamoto, K. Moriwaki, and S. Suzuki, "Fabrication of $64 \times 64$ arrayed-waveguide grating multiplexer on silicon," Electron. Lett. 31(3), 184-186 (1995).

2. K. Okamoto, K. Syuto, H. Takahashi, and Y. Ohmori, "Fabrication of 128-channel arrayed-waveguide grating multiplexer with $25 \mathrm{GHz}$ channel spacing," Electron. Lett. 32(16), 1474-1475 (1996).

3. A. Kaneko, T. Goh, H. Yamada, T. Tanaka, and I. Ogawa, "Design and applications of silica-based planar lightwave circuits," IEEE J. Quantum Electron. 5(5), 1227-1236 (1999).

4. A. Himeno, K. Kato, and T. Miya, "Silica-based planar lightwave circuits," IEEE J. Sel. Top. Quantum Electron. 4(6), 913-924 (1998). 